\title{
Effect of tricuspid annuloplasty concomitant with left heart surgery on right heart geometry and function
}

Tomoki Sakata, MD, ${ }^{a}$ Kenji Mogi, MD, ${ }^{\mathrm{a}}$ Manabu Sakurai, MD, ${ }^{\mathrm{a}}$ Anan Nomura, MD, ${ }^{\mathrm{a}}$ Masahiko Fujii, MD, ${ }^{\mathrm{a}}$ Daisuke Kaneyuki, MD, ${ }^{a}$ Goro Matsumiya, MD, PhD, ${ }^{b}$ and Yoshiharu Takahara, MD $^{\mathrm{a}}$

\section{ABSTRACT}

Objectives: To elucidate the effect of tricuspid annuloplasty concomitant with left-sided valve surgery on the right heart in patients with mild or more tricuspid regurgitation $(\mathrm{TR})$.

Methods: We enrolled 78 patients with mild or more TR who underwent leftsided valve surgery. Forty-three patients underwent only left-sided valve surgery (group non-T) and 35 underwent concomitant tricuspid annuloplasty (group T). Echocardiographic changes between the preoperative and 1-year follow-up periods were compared. Propensity score matching was used to obtain riskadjusted outcome comparisons (16 pairs).

Results: In group non-T, there were more operations for aortic stenosis and concomitant coronary artery bypass grafting, and fewer operations for mitral regurgitation. The prevalence of atrial fibrillation was higher in group $\mathrm{T}$. In preoperative echocardiography, there were no significant differences in left ventricular and right ventricular (RV) dimensions and functions. Tricuspid valve annular diameter and TR-related parameters were significantly larger in group T. Left ventricular dimensions and TR-related parameters significantly improved in both groups 1 year after operation. RV diameter was significantly reduced in only group T. In analysis of variance, RV diameter in systole and diastole showed significant interaction, whereas left heart dimensions and function, tricuspid valve tethering height, and RV fractional area change did not show interaction. These results were not attenuated even after propensity-matching analyses.

Conclusions: Among patients with mild or more TR, RV reverse remodeling was not obtained with left-sided valve surgery alone. Additional use of tricuspid annuloplasty might potentially achieve favorable TR regulation as well as RV reverse remodeling. (J Thorac Cardiovasc Surg 2018;156:1050-61)

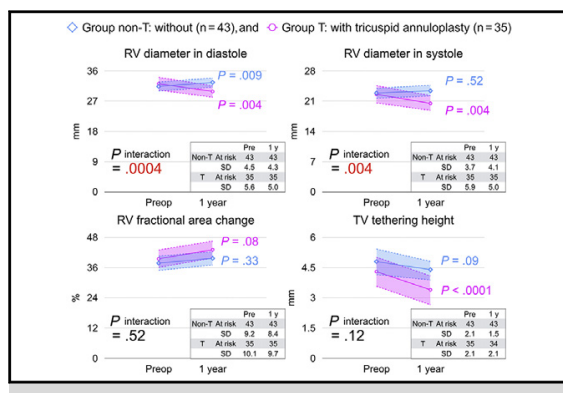

Interaction between tricuspid annuloplasty and improvement of RV dimensions and function.

\section{Central Message}

Tricuspid annuloplasty concomitant with leftsided valve surgery might potentially achieve not only favorable TR regulation but also RV reverse remodeling in patients with mild or more preoperative TR.

\section{Perspective}

We assessed the effect of concomitant tricuspid annuloplasty on the right heart in patients with mild or more TR. This study revealed that RV reverse remodeling was achieved 1 year after surgery only when tricuspid annuloplasty was also used in left-sided valve surgery, and that 1 year was not sufficient to show a significant difference in terms of RV functional recovery with additional use of tricuspid annuloplasty.

See Editorial Commentary page 1062.
Tricuspid annuloplasty concomitant with left-sided valve surgery is commonly used to control functional tricuspid regurgitation (TR) and has shown favorable outcomes in

From the a Division of Cardiovascular Surgery, Heart and Vascular Institute, Funabashi Municipal Medical Center, Funabashi, Japan; and ${ }^{\mathrm{b}}$ Department of Cardiovascular Surgery, Chiba University Hospital, Chiba, Japan.

This article was presented at Mitral Conclave 2017 Breakout Session, The American Association for Thoracic Surgery Week, April 27-28, 2017

Received for publication July 5, 2017; revisions received Jan 11, 2018; accepted for publication March 2, 2018; available ahead of print May 1, 2018.

Address for reprints: Tomoki Sakata, MD, Division of Cardiovascular Surgery, Heart and Vascular Institute, Funabashi Municipal Medical Center, 1-21-1 Kanasugi, Funabashi, Chiba 273-8588, Japan (E-mail: bpbck570@ybb.ne.jp).

0022-5223/\$36.00

Copyright (c) 2018 by The American Association for Thoracic Surgery

https://doi.org/10.1016/j.jtcvs.2018.03.101 terms of the freedom from recurrent TR. ${ }^{1-4}$ Because TR is affected by right heart dimensions, current guidelines recommend tricuspid annuloplasty for not only preoperative severe TR but also for tricuspid annular dilatation (TAD) of $>40 \mathrm{~mm} .{ }^{5,6}$ TAD is an ongoing process, ${ }^{1}$ and associated with postoperative right ventricular (RV) remodeling. It subsequently leads to right heart failure

- Scanning this $\mathrm{QR}$ code will take you to a supplemental video for the article. 


$$
\begin{aligned}
& \text { Abbreviations and Acronyms } \\
& \begin{aligned}
\mathrm{LV} & =\text { left ventricular } \\
\mathrm{RA} & =\text { right atrial } \\
\mathrm{RV} & =\text { right ventricular } \\
\mathrm{TAD} & =\text { tricuspid annular dilatation } \\
\mathrm{TR} & =\text { tricuspid regurgitation }
\end{aligned}
\end{aligned}
$$

if preoperative TR remains untreated during primary surgery. Because the presence of a "synergistic relationship" between TR and RV dysfunction has been gradually made clear, ${ }^{7,8}$ the tricuspid valve has, more than ever, become the focus of investigations. Recent reports showed that tricuspid annuloplasty can promote improvement of RV function evaluated with several parameters, including RV diameter, tricuspid annular plane systolic excursion, and pulmonary artery pressure, in 2- to 5-year follow-up periods after surgery. ${ }^{1,3,9}$ The aim of this study was to elucidate the effect of tricuspid annuloplasty concomitant with left-sided valve surgery on right heart geometry and function for up to 1 year after the surgery.

\section{METHODS \\ Study Population}

With permission from our institutional review board (approval number 29-6; date of approval: June 26, 2017), we retrospectively reviewed patient data. The need for individual patient consent was waived. From January 2012 to March 2016, 106 patients with mild or more TR underwent left-sided valve surgery. Among them, patients with primary TR, active infective endocarditis, previous pacemaker lead implantation, and missing data were excluded, leaving 78 patients enrolled. Patients with missing data were postoperatively followed-up at other hospitals from which the patients were originally referred for surgery. Therefore we could not obtain the follow-up echocardiographic data. The cohort was divided into 2 groups: group non-T included 43 patients who underwent only left-sided valve surgery, and group $\mathrm{T}$ included 35 patients who underwent left-sided valve surgery and tricuspid annuloplasty (Figure 1). Because some preoperative characteristics were significantly different between groups, propensity score matching was used to enable more balanced comparisons. The end point of this study was the improvement of each echocardiographic parameter between the preoperative and 1 year follow-up periods.

\section{Data Collection and Assessment of Echocardiographic Parameters}

Patient characteristics, surgical, and in-hospital outcomes, and followup data were extracted from hospital electronic medical records. Preoperative and 1 year follow-up transthoracic echocardiography data were available for all 78 patients. Left heart dimensions and function, right heart dimensions and function, and parameters of TR including pressure gradient, TR jet area, right atrial (RA) area, and their ratio were assessed. To analyze TR severity as a continuous datum, we defined it as the ratio of the TR jet area to the RA area. In this study, trivial TR $(1+)$ corresponds to a ratio of $<0.1$, mild $(2+)$ between 0.1 and 0.2 , moderate $(3+)$ between 0.2 and 0.33 , and severe $(4+)>0.33$.

Right heart dimensions and quantitative TR data were not routinely measured in all patients in our institute. Therefore, echocardiographic data in all 78 patients were remeasured retrospectively by 2 expert

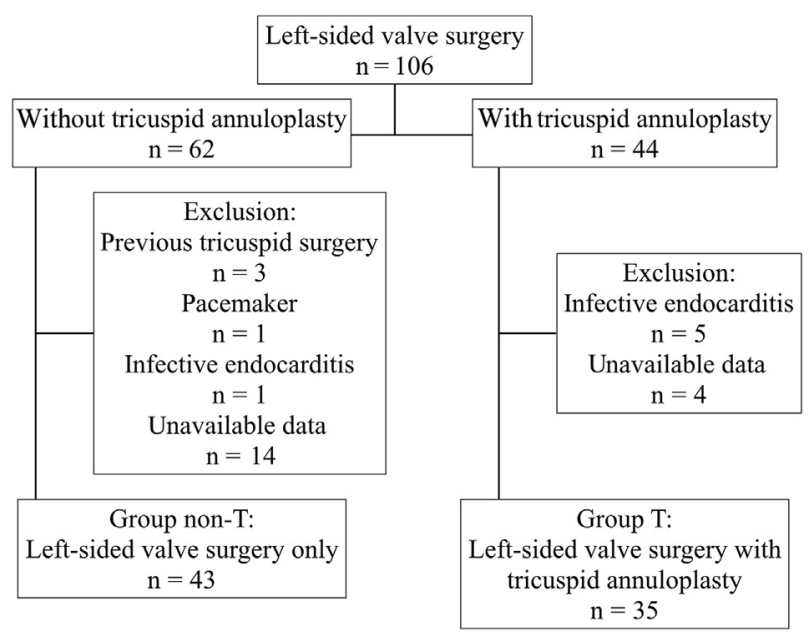

FIGURE 1. Flow chart for patient selection.

echocardiographers. The measurement method was standardized and on the basis of current guidelines. Right heart dimensions, function, and TR severity were obtained from a standard 4-chamber view, which was available in all patients. To ensure internal consistency of echocardiographic data measured by the 2 examiners, preoperative RV diameter in diastole was measured in all 78 patients by each examiner. The values determined by 1 examiner were plotted against those of the other on a scatter diagram (Figure 2). The plotted points were mostly distributed along the $\mathrm{Y}=\mathrm{X}$ graph.

\section{Surgical Procedure}

All surgeries were performed through a median sternotomy with standard cardiopulmonary bypass at mild hypothermia. Myocardial protection was achieved with antegrade and/or retrograde cold crystalloid cardioplegia. When patients had moderate or severe TR, we added tricuspid annuloplasty to left-sided valve surgery according to current guidelines. In addition, tricuspid annuloplasty was also considered in patients with mild TR if they had previously reported risk factors for recurrent TR, including atrial fibrillation and pulmonary hypertension.

For the 35 patients who underwent tricuspid annuloplasty, the repair was done with either the De Vega procedure $(n=5)$ or ring annuloplasty $(\mathrm{n}=30)$. We used an MC3 prosthetic ring (Edwards Lifescience Corp, Irvine, CA) of sizes 26,28 , and $30 \mathrm{~mm}$ in 13,15 , and 2 patients, respectively. We usually used a $28-\mathrm{mm}$ ring to correct the dilated annulus to the normal size; however, sometimes, other sizes were used according to the body surface area of patients. Nine- or 10-ring sutures were placed carefully to avoid injury to the conduction system (Video 1).

\section{Statistical Methods}

Categorical variables are presented as percentages and numbers, and were compared using Fisher exact test. Continuous variables are presented as median and interquartile range, and were compared using MannWhitney $U$ test. Changes of each continuous variable with time were analyzed using Wilcoxon signed rank test.

To analyze the effect of tricuspid annuloplasty on the improvement of each echocardiographic parameter between the preoperative and 1 year follow-up periods, 2-factor analysis of variance with repeated measures was conducted. If there was a significant interaction between groups and time, we considered that a parameter was affected by a group difference (whether tricuspid annuloplasty was added or not). Propensity scores were calculated for each patient using multivariate logistic regression on the basis of the following covariates: mitral valve surgery, aortic valve 


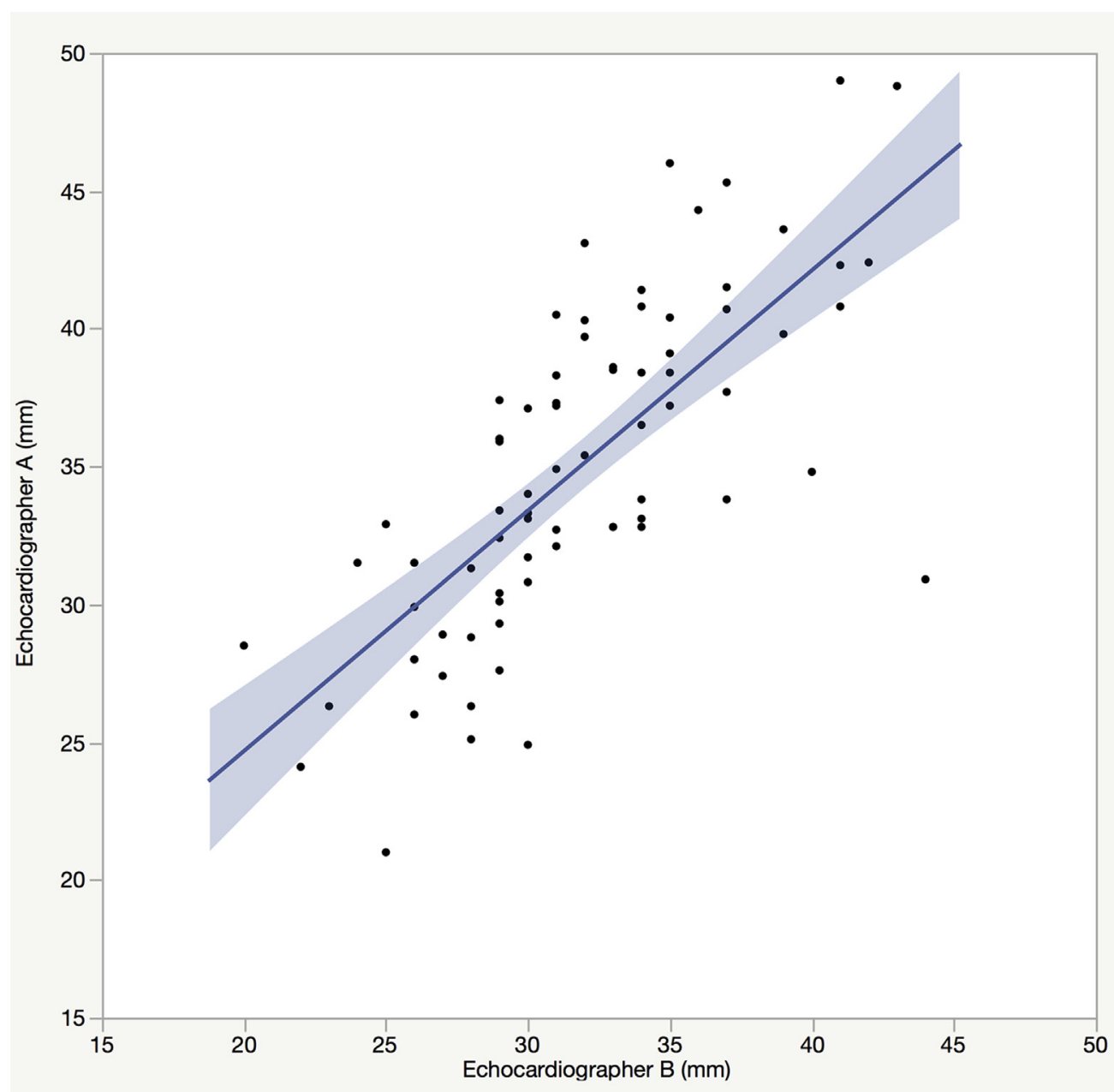

FIGURE 2. Scatter plot graph with preoperative right ventricular diameters in diastole measured using 2 echocardiographers on each axis. $\mathrm{Y}=0.872 \mathrm{X}+$ $7.23, R^{2}=0.534$.

surgery, concomitant coronary artery bypass grafting, history of atrial fibrillation, left ventricular (LV) ejection fraction, tricuspid annular diameter, TR jet area, RA area, and the TR jet area/RA area ratio. The same analyses were performed after adjustment.

All analyses were conducted with JMP version 12.0 (SAS Institute Inc, Cary, NC), and $P<.05$ was the criterion for significance. The significant level was set as $0.0042(=0.05 / 12)$ under Bonferroni correction when analysis of variance was performed. In addition to $P$ value, standardized differences were calculated and are noted in Tables 1 and 2 .

\section{RESULTS}

\section{Patient Characteristics}

Table 1 shows the preoperative and surgical characteristics for all 78 patients. Their mean age was 73 (range, 6577) years, and $44(56 \%)$ of them were women. At baseline, the prevalence of atrial fibrillation was significantly higher in group $\mathrm{T}$. There was no difference in age, sex, other medical history, or New York Heart Association classification.

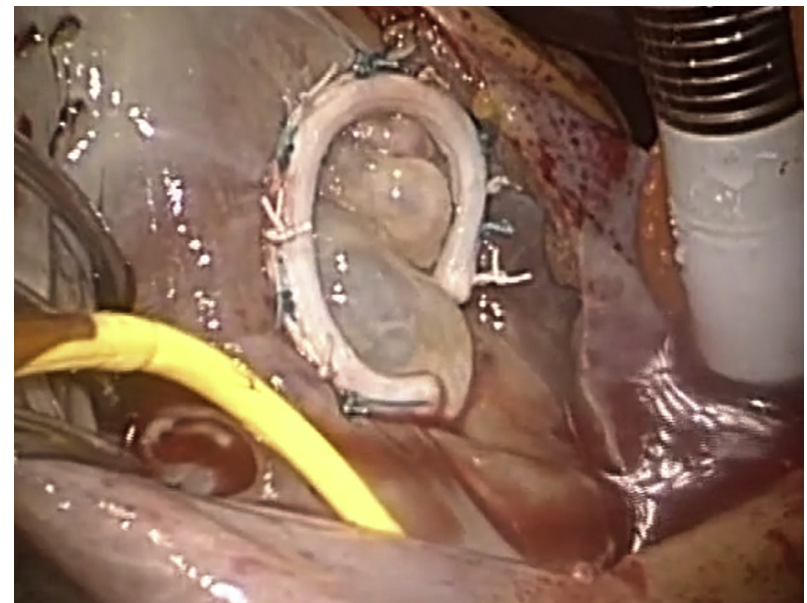

VIDEO 1. Tricuspid annuloplasty using a 28-mm MC3 ring (Edwards Lifescience Corp, Irvine, Calif). Annuloplasty stitches were placed on the annulus under cardiac arrest. Video available at: https://www.jtcvs. org/article/S0022-5223(18)30882-1/fulltext. 
TABLE 1. Patient preoperative and surgical characteristics before adjustment

\begin{tabular}{|c|c|c|c|c|c|}
\hline Characteristic & All patients $(n=78)$ & Group non-T $(n=43)$ & Group T $(n=35)$ & $P$ value & Stand. diff. \\
\hline \multicolumn{6}{|l|}{ Patient } \\
\hline Age, y (IQR) & $73(65-77)$ & $72(62-77)$ & $73(65-76)$ & .98 & 0.022 \\
\hline Female sex, $\mathrm{n}(\%)$ & $44(56)$ & $24(56)$ & $20(57)$ & 1 & 0.02 \\
\hline Body surface area, $\mathrm{m}^{2}$ (IQR) & $1.51(1.39-1.67)$ & $1.55(1.43-1.70)$ & $1.45(1.35-1.64)$ & .1 & -0.4 \\
\hline Previous cardiac surgery, $\mathrm{n}(\%)$ & $11(14)$ & $4(9)$ & $7(20)$ & .21 & 0.306 \\
\hline Atrial fibrillation, n (\%) & $32(42)$ & $10(24)$ & $22(63)$ & & \\
\hline Chronic & 29 & 9 & 20 & .001 & 0.842 \\
\hline Paroxysmal & 3 & 1 & 2 & & \\
\hline Ischemic heart disease, $\mathrm{n}(\%)$ & $14(18)$ & $9(21)$ & $5(14)$ & .56 & -0.186 \\
\hline \multicolumn{6}{|l|}{ NYHA functional class, $\mathrm{n}(\%)$} \\
\hline I & $31(40)$ & $20(48)$ & $11(31)$ & & -0.353 \\
\hline II & $33(43)$ & $18(43)$ & $15(43)$ & .11 & 0 \\
\hline III & $13(17)$ & $4(9)$ & $9(26)$ & & 0.459 \\
\hline IV & $0(0)$ & $0(0)$ & $0(0)$ & & - \\
\hline \multicolumn{6}{|l|}{ Echocardiographic data } \\
\hline LV diameter in diastole, mm (IQR) & $51(43-57)$ & $50(43-57)$ & $53(44-60)$ & .54 & 0.127 \\
\hline LV diameter in systole, mm (IQR) & $35(29-42)$ & $32(28-42)$ & $36(30-46)$ & .2 & 0.233 \\
\hline Left atrial diameter, mm (IQR) & $47(40-55)$ & $47(39-52)$ & $48(41-56)$ & .18 & 0.363 \\
\hline LV ejection fraction, \% (IQR) & $60(51-68)$ & $63(52-70)$ & $55(49-67)$ & .12 & -0.285 \\
\hline \multicolumn{6}{|l|}{ TR grade, $\mathrm{n}(\%)$} \\
\hline $1+($ none-trivial $)$ & $0(0)$ & $0(0)$ & $0(0)$ & & - \\
\hline $2+($ mild $)$ & $58(74)$ & $39(91)$ & $19(54)$ & .0005 & -0.910 \\
\hline $3+($ moderate $)$ & $19(24)$ & $4(9)$ & $15(43)$ & & 0.841 \\
\hline $4+($ severe $)$ & $1(1)$ & $0(0)$ & $1(3)$ & & 0.249 \\
\hline TR pressure gradient, mm Hg (IQR) & $38(27-46)$ & $37(26-45)$ & $41(29-50)$ & .1 & 0.39 \\
\hline Tricuspid annular diameter, mm (IQR) & $35(32-39)$ & $34(31-37)$ & $37(33-42)$ & .02 & 0.518 \\
\hline Tricuspid valve tethering height, $\mathrm{mm}$ (IQR) & $4.25(3.50-5.50)$ & $42(3.5-5.5)$ & $4.3(3.4-5.4)$ & .73 & -0.233 \\
\hline TR jet area, $\mathrm{cm}^{2}(\mathrm{IQR})$ & $3.1(2.1-5.9)$ & $2.3(1.7-3.1)$ & $52(32-6.7)$ & $<.0001$ & 0.928 \\
\hline $\mathrm{RA}$ area, $\mathrm{cm}^{2}(\mathrm{IQR})$ & $16.5(13.1-22.4)$ & $15.8(13.1-19.7)$ & $19.0(13.7-24.3)$ & .03 & 0.591 \\
\hline $\mathrm{TR}$ jet area/RA area ratio (IQR) & $0.20(0.13-0.33)$ & $0.15(0.11-0.20)$ & $0.29(0.22-0.36)$ & $<.0001$ & 1.057 \\
\hline RA diameter, mm (IQR) & $38.5(32.0-44.0)$ & $36(32-42)$ & $41(34-47)$ & .08 & 0.386 \\
\hline RV diameter in diastole, $\mathrm{mm}$ (IQR) & $31(29-35)$ & $30(29-34)$ & $32(28-35)$ & .49 & 0.165 \\
\hline RV diameter in systole, mm (IQR) & $22(19-25)$ & $23(20-25)$ & $22(18-26)$ & .35 & -0.047 \\
\hline $\mathrm{RV}$ fractional area change, $\%$ (IQR) & $38.0(32.1-45.1)$ & $37.6(29.6-45.7)$ & $38.2(33.6-44.0)$ & .4 & 0.182 \\
\hline \multicolumn{6}{|l|}{ Surgical data } \\
\hline \multicolumn{6}{|l|}{ Left valve surgery } \\
\hline Aortic stenosis, n (\%) & $15(19)$ & $12(28)$ & $3(9)$ & .04 & -0.518 \\
\hline Aortic regurgitation, $\mathrm{n}(\%)$ & $23(29)$ & $12(28)$ & $11(31)$ & .81 & 0.074 \\
\hline Mitral stenosis, n (\%) & $7(9)$ & $2(5)$ & $5(14)$ & .23 & 0.348 \\
\hline Mitral regurgitation, $\mathrm{n}(\%)$ & $49(63)$ & $22(51)$ & $27(77)$ & & \\
\hline Degenerative & 33 & 18 & 15 & .02 & 0.561 \\
\hline Functional (ischemic) & $11(4)$ & $4(3)$ & $7(1)$ & & \\
\hline Prosthetic valve failure & 5 & 0 & 5 & & \\
\hline \multicolumn{6}{|l|}{ Concomitant surgery } \\
\hline Coronary artery bypass grafting, $\mathrm{n}(\%)$ & $9(12)$ & $8(19)$ & $1(3)$ & .04 & -0.526 \\
\hline Maze, $\mathrm{n}(\%)$ & $10(13)$ & $5(12)$ & $5(14)$ & .75 & 0.08 \\
\hline Total surgery time, min (IQR) & $252(192-319)$ & $208(173-303)$ & $290(236-350)$ & .002 & 0.519 \\
\hline Cardiopulmonary bypass time, min (IQR) & $156(110-207)$ & $124(98-174)$ & $172(140-223)$ & .003 & 0.584 \\
\hline Aortic cross-clamp time, min (IQR) & $99(74-120)$ & $86(67-114)$ & $108(88-136)$ & .02 & 0.427 \\
\hline
\end{tabular}

Stand. diff., Standardized difference; $I Q R$, interquartile range; $N Y H A$, New York Heart Association; $L V$, left ventricular; $T R$, tricuspid regurgitation; $R A$, right atrial; $R V$, right ventricular.

Preoperative echocardiographic data showed no significant difference in LV parameters. Although the TR jet area, RA area, TR jet area/RA area ratio, and tricuspid annular diameter were larger in group T, the RV diameter in systole, diastole, and RV fractional area change showed no significant difference. 
TABLE 2. Patient preoperative and surgical characteristics after adjustment

\begin{tabular}{|c|c|c|c|c|c|}
\hline Characteristic & All Patients $(n=32)$ & Group non-T $(\mathrm{n}=16)$ & Group $T(n=16)$ & $P$ value & Stand. diff. \\
\hline \multicolumn{6}{|l|}{ Patient } \\
\hline Age, y (IQR) & $70(61-76)$ & $70(61-74)$ & $73(61-77)$ & .33 & 0.231 \\
\hline Female sex, n $(\%)$ & $15(47)$ & $8(59)$ & $7(44)$ & 1 & -0.124 \\
\hline Body surface area, $\mathrm{m}^{2}$ (IQR) & $1.52(1.42-1.70)$ & $1.62(1.43-1.76)$ & $1.45(1.38-1.67)$ & .16 & -0.473 \\
\hline Previous cardiac surgery, n (\%) & $3(9)$ & $1(6)$ & $2(13)$ & 1 & 0.214 \\
\hline Atrial fibrillation, $\mathrm{n}(\%)$ & $15(47)$ & $7(44)$ & $8(50)$ & & \\
\hline Chronic & 14 & 7 & 7 & 1 & 0.124 \\
\hline Paroxysmal & 1 & 0 & 1 & & \\
\hline Ischemic heart disease, $\mathrm{n}(\%)$ & $3(9)$ & $1(6)$ & $2(13)$ & 1 & 0.214 \\
\hline \multicolumn{6}{|l|}{ NYHA functional class, $\mathrm{n}(\%)$} \\
\hline I & $13(41)$ & $6(37)$ & $7(44)$ & & 0.128 \\
\hline II & $15(47)$ & $10(63)$ & $5(31)$ & .06 & -0.659 \\
\hline III & $4(12)$ & $0(0)$ & $4(25)$ & & 0.816 \\
\hline IV & $0(0)$ & $0(0)$ & $0(0)$ & & \\
\hline \multicolumn{6}{|l|}{ Echocardiographic data } \\
\hline LV diameter in diastole, mm (IQR) & $52(43-61)$ & $54(47-65)$ & $49(42-59)$ & .22 & -0.426 \\
\hline LV diameter in systole, mm (IQR) & $35(29-46)$ & $36(31-46)$ & $32(28-44)$ & .39 & -0.302 \\
\hline Left atrial diameter, mm (IQR) & $47(40-55)$ & $50(43-58)$ & $46(38-53)$ & .27 & -0.284 \\
\hline LV ejection fraction, \% (IQR) & $61(51-68)$ & $61(51-67)$ & $60(51-68)$ & .87 & -0.078 \\
\hline \multicolumn{6}{|l|}{ TR grade, n (\%) } \\
\hline $1+$ (none-trivial) & $0(0)$ & $0(0)$ & $0(0)$ & & \\
\hline $2+($ mild $)$ & $24(75)$ & $14(87)$ & $10(63)$ & .22 & -0.603 \\
\hline $3+($ moderate $)$ & $8(25)$ & $2(13)$ & $6(37)$ & & 0.603 \\
\hline $4+($ severe $)$ & $0(0)$ & $0(0)$ & $0(0)$ & & \\
\hline TR pressure gradient, $\mathrm{mm} \mathrm{Hg}(\%)$ & $5(16)$ & $37(28-45)$ & $40(26-49)$ & .76 & 0.231 \\
\hline Tricuspid annular diameter, mm (IQR) & $36(32-41)$ & $35(31-41)$ & $37(32-42)$ & .56 & 0.096 \\
\hline Tricuspid valve tethering height, $\mathrm{mm}$ (IQR) & $4.1(3.0-5.2)$ & $4.2(3.2-6.5)$ & $4.1(2.7-4.6)$ & .27 & -0.6 \\
\hline TR jet area, $\mathrm{cm}^{2}(\mathrm{IQR})$ & $3.2(2.4-4.8)$ & $2.7(2.1-4.1)$ & $3.9(3.0-5.1)$ & .11 & 0.379 \\
\hline $\mathrm{RA}$ area, $\mathrm{cm}^{2}(\mathrm{IQR})$ & $17.0(13.1-23.0)$ & $15.6(13.2-23.0)$ & $18.5(12.5-23.8)$ & .55 & 0.237 \\
\hline TR jet area/RA area ratio (IQR) & $0.22(0.12-0.29)$ & $0.19(0.13-0.27)$ & $0.23(0.12-0.29)$ & .43 & 0.266 \\
\hline RA diameter, mm (IQR) & $40(36-46)$ & $39(36-45)$ & $41(35-47)$ & .71 & 0.06 \\
\hline $\mathrm{RV}$ diameter in diastole, $\mathrm{mm}$ (IQR) & $31(29-35)$ & $30(29-35)$ & $32(28-34)$ & .61 & 0.129 \\
\hline RV diameter in systole, mm (IQR) & $22(20-25)$ & $24(20-26)$ & $22(18-25)$ & .14 & -0.393 \\
\hline $\mathrm{RV}$ fractional area change, $\%$ (IQR) & $38.1(30.4-42.8)$ & $33.1(25.1-44.3)$ & $38.3(33.8-41.2)$ & .29 & 0.367 \\
\hline \multicolumn{6}{|l|}{ Surgical data } \\
\hline \multicolumn{6}{|l|}{ Left valve surgery } \\
\hline Aortic stenosis, n (\%) & $3(9)$ & $2(13)$ & $1(6)$ & 1 & -0.214 \\
\hline Aortic regurgitation, $\mathrm{n}(\%)$ & $9(28)$ & $3(19)$ & $6(38)$ & .43 & 0.425 \\
\hline Mitral stenosis, n (\%) & $2(6)$ & $2(13)$ & $0(0)$ & .48 & -0.535 \\
\hline Mitral regurgitation, $\mathrm{n}(\%)$ & $20(63)$ & $11(69)$ & $9(56)$ & & \\
\hline Degenerative & 13 & 9 & 4 & .72 & -0.26 \\
\hline Functional (ischemic) & $5(1)$ & $2(1)$ & $3(0)$ & & \\
\hline Prosthetic valve failure & 2 & 0 & 2 & & \\
\hline \multicolumn{6}{|l|}{ Concomitant surgery } \\
\hline Coronary artery bypass grafting, $\mathrm{n}(\%)$ & $2(6)$ & $1(6)$ & $1(6)$ & 1 & 0 \\
\hline Maze, n (\%) & $5(16)$ & $4(25)$ & $1(6)$ & .33 & -0.535 \\
\hline Total surgery time, min (IQR) & $260(202-317)$ & $227(185-305)$ & $284(222-327)$ & .12 & 0.653 \\
\hline Cardiopulmonary bypass time, $\min (\mathrm{IQR})$ & $162(123-198)$ & $140(115-185)$ & $167(139-216)$ & .19 & 0.418 \\
\hline Aortic cross-clamp time, min (IQR) & $102(79-118)$ & $93(74-115)$ & $108(90-121)$ & .26 & 0.311 \\
\hline
\end{tabular}

Stand. diff., Standardized difference; $I Q R$, interquartile range; $N Y H A$, New York Heart Association; $L V$, left ventricular; $T R$, tricuspid regurgitation; $R A$, right atrial; $R V$, right ventricular.

In group non-T, there were more surgeries for aortic stenosis and concomitant coronary artery bypass grafting, and fewer surgeries for mitral regurgitation. The surgical time and cardiopulmonary bypass time were longer in group T. No patient required postoperative pacemaker implantation in this cohort. 
TABLE 3. One year follow-up echocardiographic data before adjustment

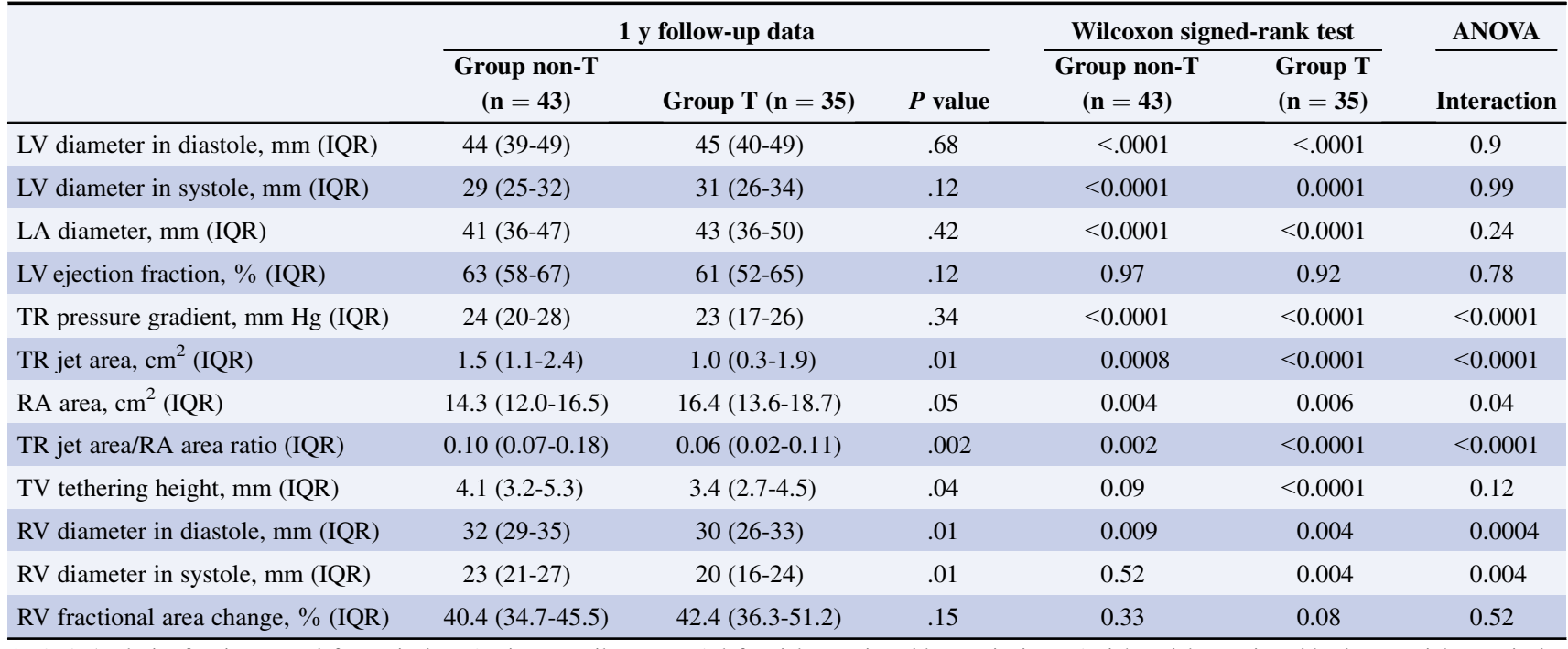

$A N O V A$, Analysis of variance; $L V$, left ventricular; $I Q R$, interquartile range; $L A$, left atrial; $T R$, tricuspid regurgitation; $R A$, right atrial; $T V$, tricuspid valve; $R V$, right ventricular.

\section{Changes of Echocardiographic Data and Effect of Tricuspid Annuloplasty on Them}

Table 3 shows echocardiographic data at 1 year followup, the results of Wilcoxon signed rank test, and interaction. Figure 3 shows the improvement of each echocardiographic parameter between the preoperative and 1-year follow-up periods, according to group. The results of Wilcoxon signed rank test are shown in the same colors as graphs. In both groups, TR severity evaluated according to the TR jet area/RA area ratio was reduced significantly at 1 year after surgery. In terms of TR grade, the TR severity of group non$\mathrm{T}$ remained mild whereas that of group $\mathrm{T}$ changed from moderate to trivial. The RV diameter in diastole and systole showed different changes according to group: that of group non-T showed a tendency to increase, whereas that of group $\mathrm{T}$ significantly decreased over time.

The results of 2-factor analysis of variance with repeated measures on echocardiographic parameters are also shown in Figure 3. First, the left heart dimensions including LV diameter in systole and diastole, left atrial diameter, and LV ejection fraction did not show significant interaction. Second, the parameters of TR, including pressure gradient, jet area, and their ratio showed significant interaction. Finally, concerning RV parameters, tricuspid valve tethering height and RV fractional area change did not show interaction. On the contrary, the RV diameter in systole and diastole showed significant interaction.

\section{Propensity Score Matching}

Because of the retrospective nature of the study, there were some differences in patient characteristics. We examined how each difference in background affected our results. The sample size was too small to conduct subgroup analysis for each factor. Therefore, we conducted propensity score matched analyses using covariates that are clinically important (mitral valve surgery, aortic valve surgery, concomitant coronary artery bypass grafting, history of atrial fibrillation, LV ejection fraction, TR jet area, RA area, and the TR jet area/RA area ratio), yielding 16 matched pairs. The preoperative characteristics after adjustment are shown in Table 2. There was no significant difference between groups. The 1-year follow-up data, the results of the Wilcoxon signed rank test between preoperative and 1-year follow-up periods, and interaction are shown in Table 4. Figure 4 shows the improvement of each echocardiographic parameter between the preoperative and 1-year follow-up periods, according to groups. The significant interactions between time and tricuspid annuloplasty were seen in RV diameter even after adjustment (in diastole: $P=.0008$, in systole: $P=.001$ ).

\section{DISCUSSION}

The main findings of this study was that tricuspid annuloplasty concomitant with left-sided valve surgery had a positive effect on the improvement of postoperative RV geometry 1 year after surgery, whereas RV remodeling was observed in patients who underwent only left-sided valve surgery. Tricuspid annuloplasty did not show a significant effect on the improvement of RV function 1 year after surgery. The same results were also seen after propensity score matched analyses. In most previous reports regarding the recovery of RV dimensions and function, tricuspid annuloplasty was performed concomitantly with mitral valve surgery. However, multivariate analysis 
$\diamond$ Group non-T: without $(n=43)$, and - Group T: with tricuspid annuloplasty $(n=35)$

LV diameter in diastole

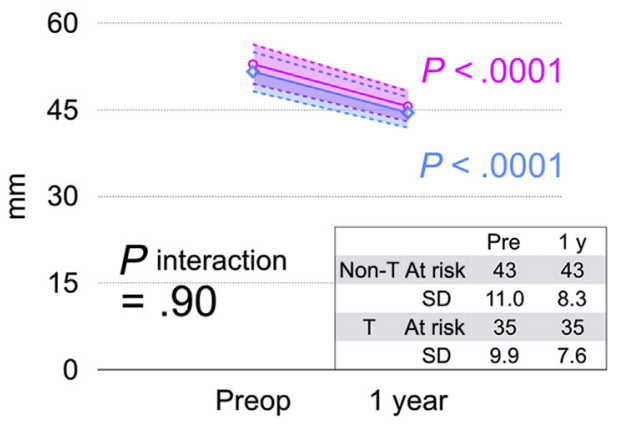

Left atrial diameter

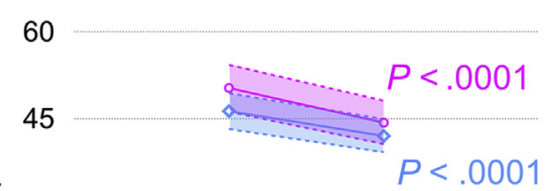

$\stackrel{\varepsilon}{\varepsilon} 30$

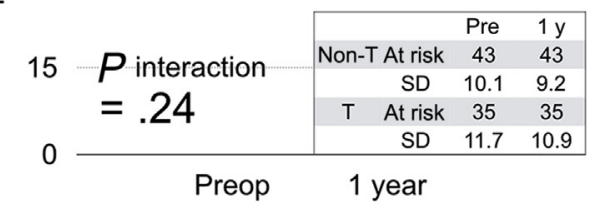

LV diameter in systole

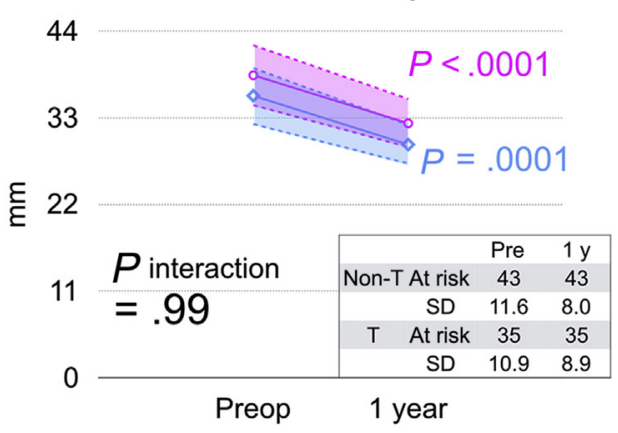

LV ejection fraction

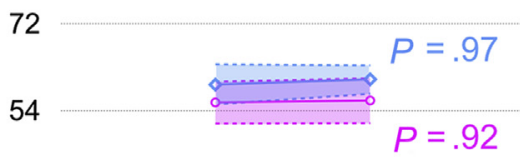

ஃ̊ 36

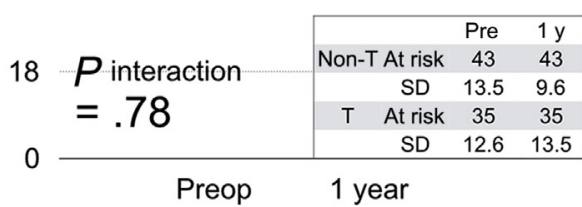

Group non-T: without $(n=43)$, and - - Group T: with tricuspid annuloplasty $(n=35)$

TR pressure gradient

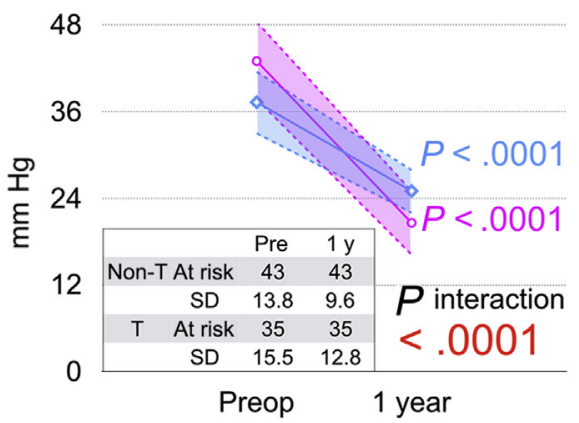

RA area

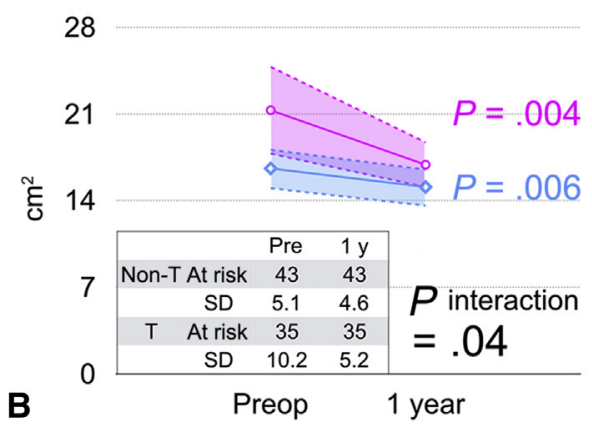

TR jet area

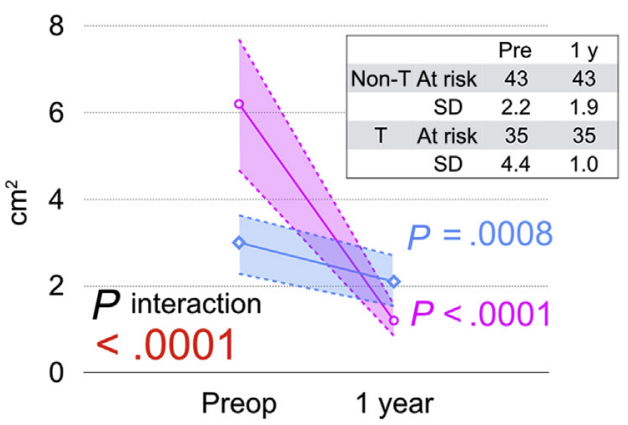

TR jet area / RA area ratio

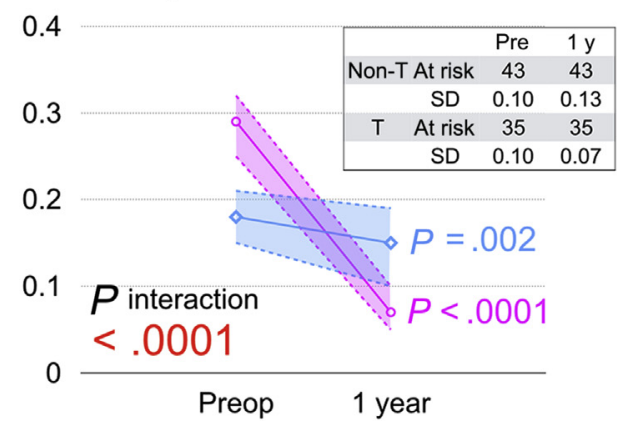

FIGURE 3. The effect of tricuspid annuloplasty concomitant with left-sided valve surgery on each echocardiographic parameter calculated using 2-factor analysis of variance with repeated measures. The results of Wilcoxon signed rank test are shown in the same colors. A, The results for the left heart dimensions and function. B, The results (only Wilcoxon signed rank test) for the parameters regarding tricuspid regurgitation (TR). C, The results for the right heart dimensions and function. $L V$, Left ventricular; Pre, preoperative; $R A$, right atrial; $R V$, right ventricular; $T V$, tricuspid valve. 
Group non-T: without $(n=43)$, and $-O$-Group T: with tricuspid annuloplasty $(n=35)$

$\mathrm{RV}$ diameter in diastole

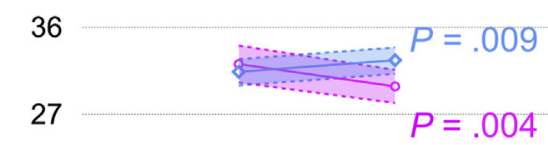

$\xi 18$

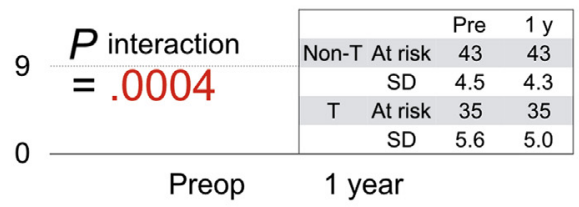

$\mathrm{RV}$ fractional area change

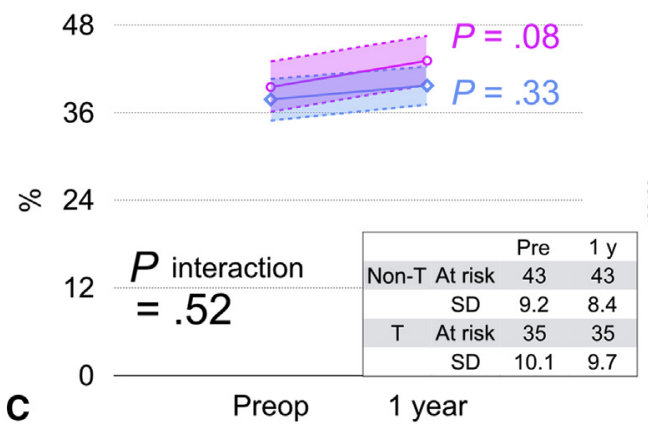

RV diameter in systole

28

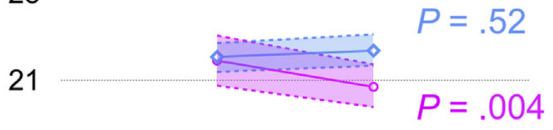

$\stackrel{\xi}{\varepsilon} 14$

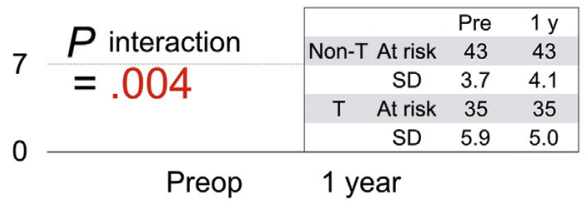

TV tethering height

6

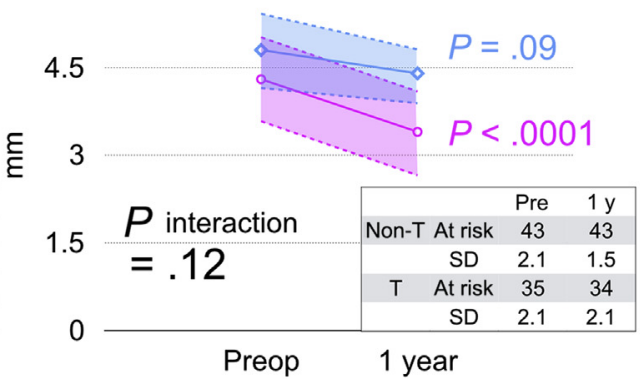

FIGURE 3. (Continued).

showed that tricuspid annuloplasty had beneficial effects on RV reverse remodeling regardless of the type of leftsided valve disease, LV ejection fraction, presence of atrial fibrillation, and concomitant coronary artery bypass grafting. In addition, we found that RV reverse remodeling after tricuspid annuloplasty can be seen at
1 year postoperatively, whereas RV remodeling progressed in patients who did not undergo tricuspid annuloplasty. These findings are new to the literature, and support an aggressive approach to perform tricuspid annuloplasty before TR becomes more complicated, with leaflet tethering caused by RV dilatation.

TABLE 4. One year follow-up echocardiographic data after adjustment

\begin{tabular}{|c|c|c|c|c|c|c|}
\hline & \multicolumn{3}{|c|}{$1 \mathrm{y}$ follow-up data } & \multicolumn{2}{|c|}{ Wilcoxon signed rank test } & \multirow{2}{*}{$\begin{array}{c}\text { ANOVA } \\
\text { Interaction }\end{array}$} \\
\hline & $\begin{array}{l}\text { Group non-T } \\
\quad(\mathrm{n}=16)\end{array}$ & $\begin{array}{l}\text { Group T } \\
(\mathbf{n}=16)\end{array}$ & $P$ value & $\begin{array}{l}\text { Group non- } T \\
\quad(\mathrm{n}=16)\end{array}$ & $\begin{array}{l}\text { Group T } \\
(\mathrm{n}=16)\end{array}$ & \\
\hline LV diameter in diastole, $\mathrm{mm}$ (IQR) & $46(39-50)$ & $42(39-48)$ & .69 & 0.0003 & 0.002 & 0.16 \\
\hline LV diameter in systole, mm (IQR) & $30(26-34)$ & $29(26-33)$ & .84 & 0.001 & 0.007 & 0.29 \\
\hline LA diameter, mm (IQR) & $41(36-51)$ & $39(35-45)$ & .37 & 0.007 & 0.003 & 0.78 \\
\hline LV ejection fraction, $\%$ (IQR) & $61(56-65)$ & $61(54-67)$ & .88 & 0.96 & 0.7 & 0.55 \\
\hline TR pressure gradient, mm Hg (IQR) & $24(19-28)$ & $23(0-28)$ & .7 & 0.001 & 0.002 & 0.35 \\
\hline TR jet area, $\mathrm{cm}^{2}(\mathrm{IQR})$ & $1.7(1.0-2.4)$ & $1.0(0.05-1.88)$ & .03 & 0.01 & $<.0001$ & 0.02 \\
\hline $\mathrm{RA}$ area, $\mathrm{cm}^{2}(\mathrm{IQR})$ & $14.3(11.5-17.0)$ & $15.9(12.4-18.2)$ & .56 & 0.03 & 0.06 & 0.57 \\
\hline TR jet area/RA area ratio (IQR) & $0.11(0.07-0.18)$ & $0.07(0.00-0.11)$ & .01 & 0.02 & $<.0001$ & 0.02 \\
\hline TV tethering height, mm (IQR) & $3.9(3.2-5.5)$ & $3.6(2.7-4.3)$ & .16 & 0.65 & 0.18 & 0.83 \\
\hline $\mathrm{RV}$ diameter in diastole, $\mathrm{mm}$ (IQR) & $32(28-37)$ & $28(26-32)$ & .03 & 0.07 & 0.007 & 0.0008 \\
\hline RV diameter in systole, mm (IQR) & $21(25-27)$ & $16(18-24)$ & .003 & 0.09 & 0.005 & 0.001 \\
\hline RV fractional area change, $\%$ (IQR) & $39.1(31.1-42.7)$ & $47.7(39.1-53.2)$ & .01 & 0.53 & 0.01 & 0.21 \\
\hline
\end{tabular}

ANOVA, Analysis of variance; $I Q R$, interquartile range; $L V$, left ventricular; $L A$, left atrial; $T R$, tricuspid regurgitation; $R A$, right atrial; $T V$, tricuspid valve; $R V$, right ventricular. 
$\diamond$ Group non-T: without $(\mathrm{n}=16)$, and $-\mathrm{O}$-Group T: with tricuspid annuloplasty $(\mathrm{n}=16)$

LV diameter in diastole

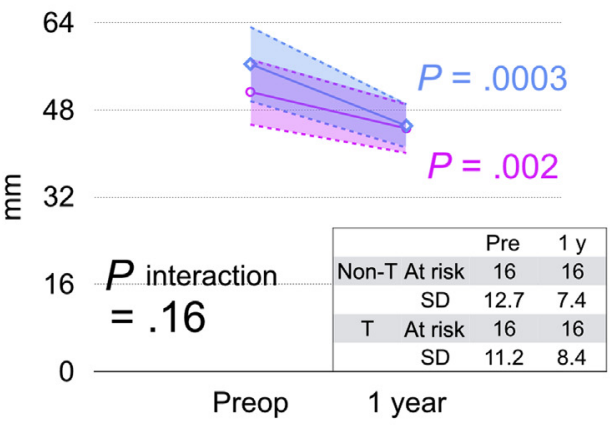

Left atrial diameter

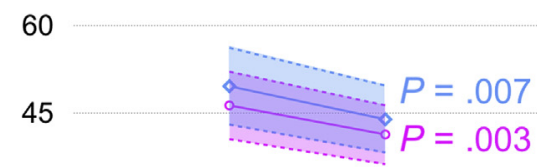

દ้ 30

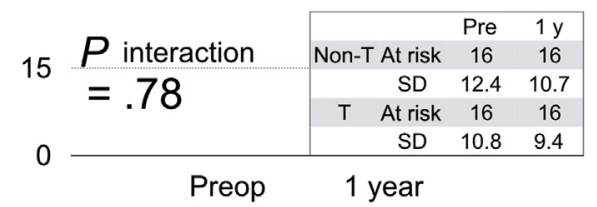

LV diameter in systole

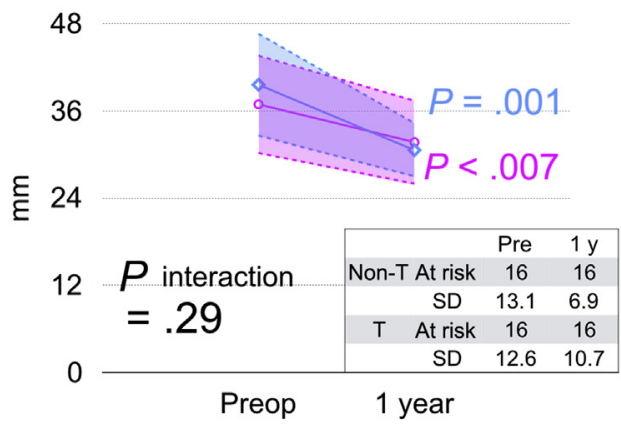

LV ejection fraction

72

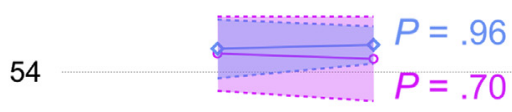

ஃ 36

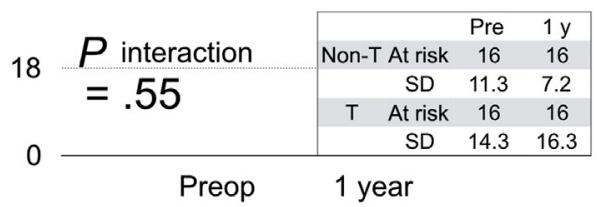

Group non-T: without $(n=16)$, and -O- Group T: with tricuspid annuloplasty $(n=16)$

TR pressure gradient

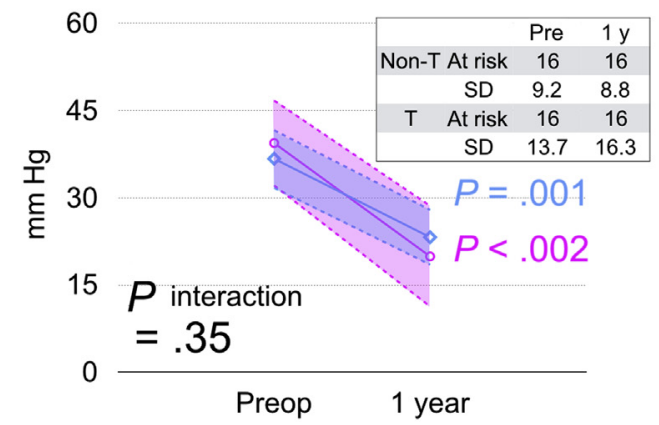

RA area

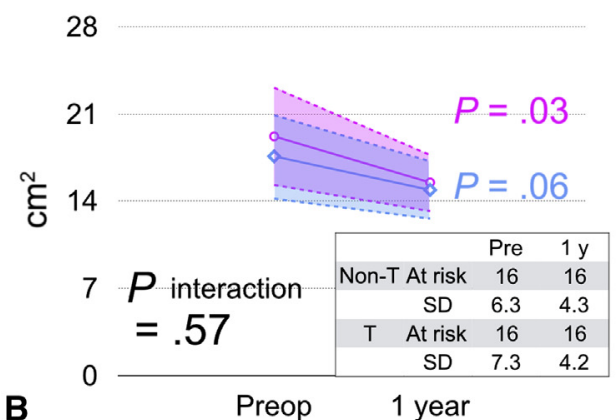

TR jet area

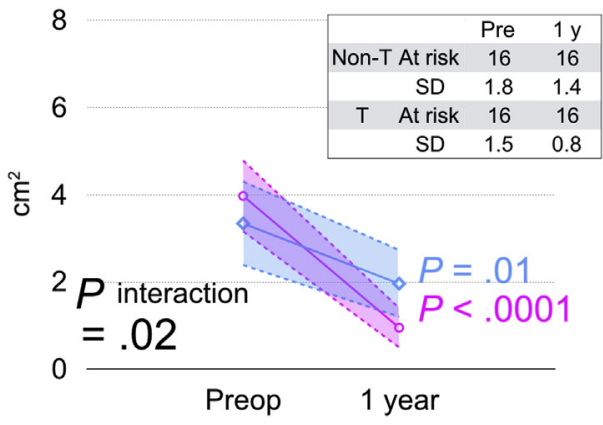

TR jet area / RA area ratio

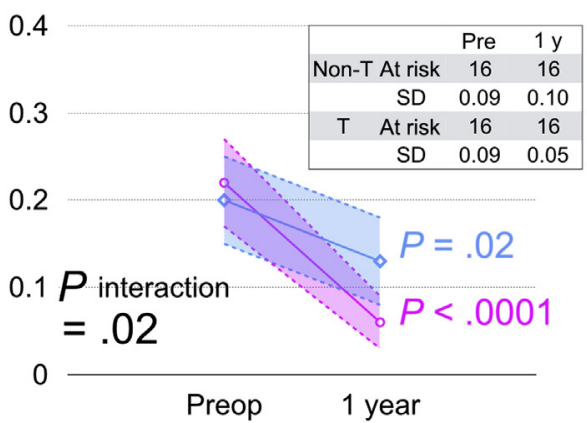

FIGURE 4. Echocardiographic changes after adjustment for preoperative and surgical differences using propensity score matching (16 pairs). A, The results for the left heart dimensions and function. B, The results for the parameters regarding tricuspid regurgitation (TR). $\mathrm{C}$, The results for the right heart dimensions and function. $L V$, Left ventricular; Pre, preoperative; $R A$, right atrial; $R V$, right ventricular; $T V$, tricuspid valve. 
$\diamond$ Group non-T: without $(n=16)$, and-O-Group T: with tricuspid annuloplasty $(n=16)$
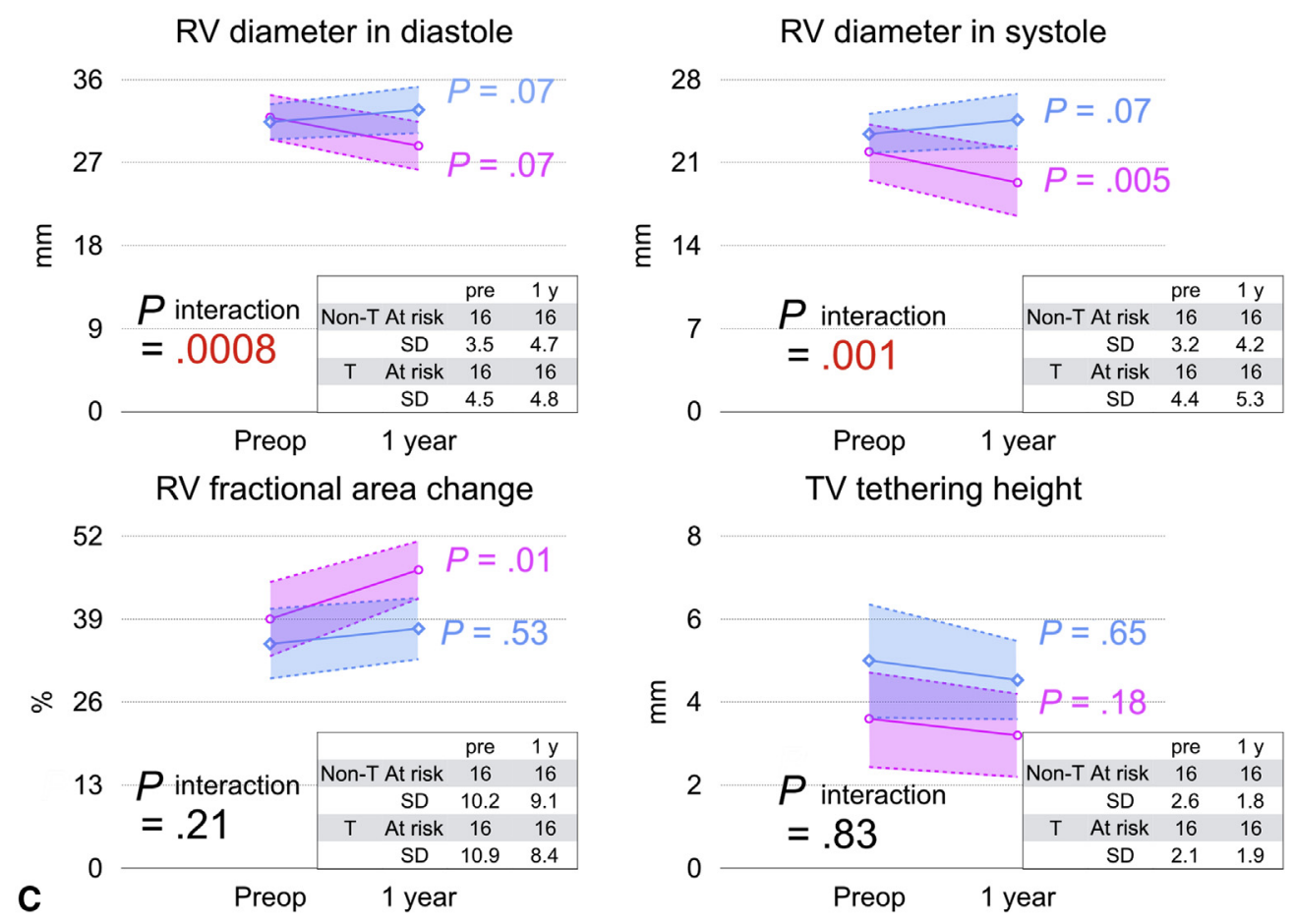

FIGURE 4. (Continued).

Clinicians have been conservative in performing tricuspid annuloplasty concomitant with left-sided valve surgery, in particular with mitral valve surgery, because some reports showed that mitral valve surgery alone could reduce TR. ${ }^{10,11}$ That belief has gradually changed, because many authors revealed a high incidence of recurrent and progressive TR. ${ }^{12-15}$ Other authors also showed that postoperative TR affects long-term outcomes, including mortality and morbidity, ${ }^{14,16-21}$ and current guidelines have shown a more aggressive attitude toward additional tricuspid annuloplasty. This attitude is supported by the facts that additional use of tricuspid annuloplasty positively affects the mid- and long-term outcomes including survival ${ }^{1,13,14,22-24}$ and that it does not increase surgical risks, ${ }^{2,18,25-27}$ whereas reoperation in patients with severe TR which was untreated in the previous left-sided valve surgery has high mortality. ${ }^{5,18,28,29}$ Furthermore, recent reports suggested that eliminating preoperative TR has a positive effect on improving RV dimensions and function, and on controlling recurrent $\mathrm{TR}^{3,8,9}$ Van de Veire et al reported that mitral valve surgery alone in patients with mild or less TR but with TAD caused progressive TR, increased TR pressure gradient, and RV remodeling postoperatively, whereas tricuspid annuloplasty concomitant with mitral valve surgery in patients with significant TR or TAD achieved $\mathrm{RV}$ reverse remodeling as well as reduction of TR and pressure gradient at 2-year follow-up. ${ }^{1}$ In our study, similar results were obtained with regard to RV reverse remodeling at 1 year after surgery, which shows that the geometric improvement of the right ventricle starts from the early phase of the postoperative course. TR did not progress regardless of tricuspid annuloplasty. However, TR was controlled at the level of less than trivial in group T, whereas TR remained mild in group non-T. This finding explains the reason for the differences in geometric improvement during the time course. The volume overload caused by TR affects the right ventricle differently, although the preoperative RV diameter was almost the same. After releasing volume overload postoperatively, the RV diameter was reduced only in group $\mathrm{T}$. This difference was produced because the right ventricle is a volume-dependent chamber. Nevertheless, we agree that medical treatment using diuretics is a reasonable alternative to control volume overload as long as TR is stably maintained at mild or less severity.

Chikwe et al compared patients who underwent mitral valve repair alone with those who underwent mitral valve repair and tricuspid annuloplasty. ${ }^{9}$ They revealed that additional use of tricuspid annuloplasty induced improvement of RV function. The proportion of patients with RV dysfunction became almost the same between the 2 groups at 3 to 5 years after surgery, although that proportion was significantly higher in patients with mitral valve repair alone preoperatively. Desai et al also reported the 
continuous improvement of RV function in patients with $3+$ or $4+$ TR if tricuspid annuloplasty was performed concomitantly with mitral valve repair. ${ }^{3}$ This effect persisted, and RV function recovered up to almost the normal level at 3 years after surgery. This finding showed that RV function is reversible.

From these previous reports and the results of this study, our speculation for the recovery of RV dimensions and function is that, after relieving pressure overload through left-sided valve surgery and volume overload through tricuspid annuloplasty, the RV dimensions can be improved and TR regulation can be maintained for 1 year. This might lead to the improvement of RV function thereafter.

\section{Study Limitation}

This study is limited by its retrospective and observational nature, as well as the small sample size. Because echocardiographic data were reviewed retrospectively, the readers were not blinded to what procedures had been performed. In the patient selection, 18 patients were excluded because they were followed-up in other hospitals. This exclusion due to unavailability of follow-up data might potentially lead to selection bias.

To assess the effects of small sample size and differences in background, we performed propensity score matched analyses; the results ensured that our speculation was valid from the viewpoint of statistical methodology. However, we cannot assess unknown confounding factors or generalize the results of this study on the basis of only 78 patients. Moreover, propensity score matching produced only 16 pairs. The disappearance of significant differences in preoperative characteristics after propensity score matching might be because of its smaller sample size. Another limitation is that we could not reveal the association between $\mathrm{RV}$ reverse remodeling and clinical outcomes because of the small sample size and short follow-up period.

Nevertheless, the findings of this study strongly support the benefit of tricuspid annuloplasty concomitant with left-sided valve surgery. A prospective multicenter randomized control trial is necessary to update the recommendations of current guidelines.

\section{CONCLUSIONS}

Among patients with mild or more preoperative TR, RV reverse remodeling was not achieved with left-sided valve surgery alone 1 year after surgery. Tricuspid annuloplasty concomitant with left-sided valve surgery might potentially achieve not only favorable TR regulation but also RV reverse remodeling.

\section{Conflict of Interest Statement}

Authors have nothing to disclose with regard to commercial support.

\section{References}

1. Van de Veire NR, Braun J, Delgado V, Versteegh MI, Dion RA, Klautz RJ, et al. Tricuspid annuloplasty prevents right ventricular dilatation and progression of tricuspid regurgitation in patients with tricuspid annular dilatation undergoing mitral valve repair. J Thorac Cardiovasc Surg. 2011;141:1431-9.

2. Lee H, Sung K, Kim WS, Lee YT, Park SJ, Carriere KC, et al. Clinical and hemodynamic influences of prophylactic tricuspid annuloplasty in mechanical mitral valve replacement. J Thorac Cardiovasc Surg. 2016;151:788-95.

3. Desai RR, Vargas Abello LM, Klein AL, Marwick TH, Krasuski RA, Ye Y, et al. Tricuspid regurgitation and right ventricular function after mitral valve surgery with or without concomitant tricuspid valve procedure. J Thorac Cardiovasc Surg. 2013;146:1126-32.

4. Ye Y, Desai R, Vargas Abello LM, Rajeswaran J, Klein AL, Blackstone EH, et al. Effects of right ventricular morphology and function on outcomes of patients with degenerative mitral valve disease. J Thorac Cardiovasc Surg. 2014;148: 2012-20.

5. Nishimura RA, Otto CM, Bonow RO, Carabello BA, Erwin JP III, Guyton RA, et al. 2014 AHA/ACC guideline for the management of patients with valvular heart disease: a report of the American College of Cardiology/American Heart Association task force on practice guidelines. Circulation. 2014;148:e1-132.

6. Vahanian A, Alfieri O, Andreotti F, Antunes MJ, Baron-Esquivias G, Baumgartner $\mathrm{H}$, et al. Guidelines on the management of valvular heart disease (version 2012): the joint task force on the management of valvular heart disease of the European Society of Cardiology (ESC) and the European Association for Cardio-Thoracic Surgery (EACTS). Eur J Cardiothorac Surg. 2012;42: S1-44.

7. Goldstone AB, Howard JL, Cohen JE, MacArthur JW Jr, Atluri P, Kirkpatrick JN, et al. Natural history of coexistent tricuspid regurgitation in patients with degenerative mitral valve disease: implications for future guidelines. J Thorac Cardiovasc Surg. 2014;148:2802-9.

8. Vargas Abello LM, Klein AL, Marwick TH, Nowicki ER, Rajeswaran J, Puwanant $S$, et al. Understanding right ventricular dysfunction and functional tricuspid regurgitation accompanying mitral valve disease. J Thorac Cardiovasc Surg. 2013;145:1234-41.

9. Chikwe J, Itagaki S, Anyanwu A, Adams DH. Impact of concomitant tricuspid annuloplasty on tricuspid regurgitation, right ventricular function, and pulmonary artery hypertension after repair of mitral valve prolapse. J Am Coll Cardiol. 2015;65:1931-8.

10. Braunwald NS, Ross J, Morrow AG. Conservative management of tricuspid regurgitation in patients undergoing mitral valve replacement. Circulation. 1967;35(Suppl I):I63-9.

11. Yilmaz O, Suri RM, Dearani JA, Sundt TM III, Daly RC, Burkhart HM, et al. Functional tricuspid regurgitation at the time of mitral valve repair for degenerative leaflet prolapse: the case for a selective approach. J Thorac Cardiovasc Surg. 2011;142:608-13.

12. Song H, Chung CH, Choo SJ, Song MG, Song JM, Kang DH, et al. Factors associated with development of late significant tricuspid regurgitation after successful left-sided valve surgery. Heart. 2009;95:931-6.

13. Dreyfus GD, Corbi PJ, Chan J, Bahrami T. Secondary tricuspid regurgitation or dilatation: which should be the criteria for surgical repair? Ann Thorac Surg. 2005;79:127-32.

14. Calafiore AM, Gallina S, Iacò AL, Contini M, Bivona A, Gagliardi M, et al. Mitral valve surgery for functional mitral regurgitation: should moderate-ormore tricuspid regurgitation be treated? A propensity score analysis. Ann Thorac Surg. 2009;87:698-703.

15. Kwak JJ, Kim YJ, Kim MK, Kim HK, Park JS, Kim KH, et al. Development of tricuspid regurgitation late after left-sided valve surgery: a single center experience with long-term echocardiographic examinations. Am Heart J. 2008;155: 732-7.

16. Matsunaga A, Duran CM. Progression of tricuspid regurgitation after repaired functional ischemic mitral regurgitation. Circulation. 2005;112(Suppl I):I453-7.

17. Sagie A, Schwammenthal E, Padial LR, Vazquez de Prada JA, Weyman AE, Levine RA. Determinants of functional tricuspid regurgitation in incomplete tricuspid valve closure: Doppler color flow study of 109 patients. J Am Coll Cardiol. 1994;24:446-53.

18. McCarthy PM, Bhudia SK, Rajeswaran J, Hoercher KJ, Lytle BW, Cosgrove DM, et al. Tricuspid valve repair: durability and risk factors for failure. J Thorac Cardiovasc Surg. 2004;127:674-85.

19. Anyanwu AC, Chikwe J, Adams DH. Tricuspid valve repair for treatment and prevention of secondary tricuspid regurgitation in patients undergoing mitral valve surgery. Curr Cardiol Rep. 2008;10:110-7. 
20. Jeong DS, Sung K, Kim WS, Lee YT, Yang JH, Jun TG, et al. Fate of functional tricuspid regurgitation in aortic stenosis after aortic valve replacement. J Thorac Cardiovasc Surg. 2014;148:1328-33.

21. Katsi V, Raftopoulos L, Aggeli C, Vlasseros I, Felekos I, Tousoulis D, et al. Tricuspid regurgitation after successful mitral valve surgery. Interact Cardiovasc Thorac Surg. 2012;15:102-8.

22. Gosev I, Yammine M, McGurk S, Ejiofor JI, Norman A, Ivkovic V, et al. Should moderate-to-severe tricuspid regurgitation be repaired during reoperative left-sided valve procedures? Semin Thorac Cardiovasc Surg. 2016;28: $38-45$.

23. Kim JB, Yoo DG, Kim GS, Song H, Jung SH, Choo SJ, et al. Mild-to-moderate functional tricuspid regurgitation in patients undergoing valve replacement for rheumatic mitral disease: the influence of tricuspid valve repair on clinical and echocardiographic outcomes. Heart. 2012;98:24-30.

24. Navia JL, Nowicki ER, Blackstone EH, Brozzi NA, Nento DE, Atik FA, et al. Surgical management of secondary tricuspid valve regurgitation: annulus, commissure, or leaflet procedure? J Thorac Cardiovasc Surg. 2010;139:1473-82.

25. Badhwar V, Rankin JS, He M, Jacobs JP, Furnary AP, Fazzalari FL, et al. Performing concomitant tricuspid valve repair at the time of mitral valve operations is not associated with increased operative mortality. Ann Thorac Surg. 2017;103: 587-93.

26. Filsoufi F, Salzberg SP, Coutu M, Adams DH. A three-dimensional ring annuloplasty for the treatment of tricuspid regurgitation. Ann Thorac Surg. 2006;81: 2273-7.

27. Benedetto U, Melina G, Angeloni E, Refice S, Roscitano A, Comito C, et al. Prophylactic tricuspid annuloplasty in patients with dilated tricuspid annulus undergoing mitral valve surgery. J Thorac Cardiovasc Surg. 2012;143:632-8.

28. Pfannmüller B, Moz M, Misfeld M, Borger MA, Funkat AK, Garbade J, et al Isolated tricuspid valve surgery in patients with previous cardiac surgery. J Thorac Cardiovasc Surg. 2013;146:841-7.

29. Kilic A, Saha-Chaudhuri P, Rankin JS, Conte JV. Trends and outcomes of tricuspid valve surgery in North America: an analysis of more than 50,000 patients from the Society of Thoracic Surgeons database. Ann Thorac Surg. 2013;96:1546-52.

Key Words: tricuspid regurgitation, tricuspid annuloplasty, right ventricular remodeling, right ventricular function 